\title{
Lifespan trajectories of relative corpus callosum thickness: regional differences and cognitive relevance.
}

\author{
Danielsen, V.M. (1), Vidal-Piñeiro, D. (1), Mowinckel, A.M. (1), Sederevicius, D. (1), Fjell, A.M. \\ $(1,2)$, Walhovd, K.B. $(1,2), \&$ Westerhausen, R. $(1,3)^{*}$ \\ (1) Center for Lifespan Changes in Brain and Cognition (LCBC), Department of Psychology, University of \\ Oslo, Norway \\ (2) Department of Radiology and Nuclear Medicine, Oslo University Hospital, Norway \\ (3) Department of Biological and Medical Psychology, University of Bergen, Norway
}

Running title: Lifespan corpus callosum trajectories

\author{
*Corresponding author \\ René Westerhausen \\ Center for Lifespan Changes in Brain and Cognition (LCBC) \\ Department of Psychology, University of Oslo, POB 1094 Blindern, 0317 Oslo, Norway \\ email: rene.westerhausen@psykologi.uio.no, Phone: (+47) 22845230
}




\begin{abstract}
The cerebral hemispheres are specialized for different cognitive functions and receive divergent information from the sensory organs, so that the interaction between the hemispheres is a crucial aspect of perception and cognition. At the same time, the major fiber tract responsible for this interaction, the corpus callosum, shows a structural development across the lifespan which is overproportional. That is, compared to changes in overall forebrain volume, the corpus callosum shows an accentuated growth during childhood, adolescence, and early adulthood, as well as pronounced decline in older age. However, this over-proportionality of growth and decline along with potential consequences for cognition, have been largely overlooked in empirical research. In the present study we systematically address the proportionality of callosal development in a large mixed crosssectional and longitudinal sample (1867 datasets from 1014 unique participants), covering the human lifespan (age range 4 to 93 years), and examine the cognitive consequences of the observed changes. Relative corpus callosum thickness was measured at 60 segments along the midsagittal surface, and lifespan trajectories were clustered to identify callosal subsections of comparable lifespan development. While confirming the expected inverted $\mathrm{u}$-shaped lifespan trajectories, we also found substantial regional variation. Compared with anterior clusters, posterior sections exhibited an accentuated growth during development which extends well into the third decade of life, and a protracted decline in older age which is delayed by about 10 years (starting mid to late $50 \mathrm{~s})$. We further showed that the observed longitudinal changes in relative thickness of the mid splenium significantly mediates age-related changes in tests assessing verbal knowledge and nonverbal visual-spatial abilities across the lifespan. In summary, we demonstrate that analyzing the proportionality of callosal growth and decline offers valuable insight into lifespan development of structural connectivity between the hemispheres, and suggests consequences for the cognitive development of perception and cognition.
\end{abstract}




\section{Introduction}

The two cerebral hemispheres receive different but complementary input from the senses and are specialized for different yet interdependent functions (Ocklenburg \& Güntürkün, 2018).

Consequently, the interaction between the hemispheres represents a central aspect of human perception and cognition (Banich, 1998; Gazzaniga, 2000; Glickstein \& Berlucchi, 2008), whereby not only information transfer (E. Genc, Bergmann, Singer, \& Kohler, 2011; Steinmann et al., 2018; Westerhausen, Gruner, Specht, \& Hugdahl, 2009) but also the coordination of processing between the hemispheres (Davis \& Cabeza, 2015; Hinkley et al., 2016; Thiel et al., 2006) can be demonstrated. The major white matter pathway supporting this interaction, is the corpus callosum, which contains axons and axon collaterals interconnecting virtually all regions of the cerebral cortex (Schmahmann \& Pandya, 2006).

However, the corpus callosum also undergoes significant structural changes across the lifespan following roughly an inverted-u-shaped trajectory. That is, a rapid maturation in early childhood with a substantial gain of axon myelination is, reflected in a significant increase in overall callosal size (Clarke, Kraftsik, Van der Loos, \& Innocenti, 1989; Rakic \& Yakovlev, 1968; Tanaka-Arakawa et al., 2015; Vannucci, Barron, \& Vannucci, 2017), which is followed by increasingly decelerating growth during later childhood and adolescence (Ansado et al., 2015; Chavarria, Sanchez, Chou, Thompson, \& Luders, 2014; S. Genc, Malpas, Ball, Silk, \& Seal, 2018; Giedd et al., 1999; Luders, Cherbuin, et al., 2010; Luders, Thompson, \& Toga, 2010; Westerhausen et al., 2016). "Peak" corpus callosum with respect to size and diffusion parameters appears to be reached during the third decade of life (Hasan et al., 2009; Lebel, Caverhill-Godkewitsch, \& Beaulieu, 2010; Prendergast et al., 2015; Pujol, Vendrell, Junque, Marti-Vilalta, \& Capdevila, 1993; Yeatman, Wandell, \& Mezer, 2014) followed by a phase of relative stability (Rauch \& Jinkins, 1994; Raz, Ghisletta, Rodrigue, Kennedy, \& Lindenberger, 2010) which turns into a decline above the age of 60 years (Doraiswamy et al., 1991; Hasan, Kamali, et al., 2008; Hou \& Pakkenberg, 2012; D. Salat, Ward, Kaye, \& Janowsky, 1997; Sullivan, Rohlfing, \& Pfefferbaum, 2010). Furthermore, both developmental (Chicoine, Proteau, \& Lassonde, 2000; Dougherty et al., 2007; Fryer et al., 2008; Hinkley et al., 2016; Westerhausen et al., 2011) and aging studies (Gootjes et al., 2006; Kennedy \& Raz, 2009; Penke et al., 2010; Prendergast et al., 2015; Zahr, Rohlfing, Pfefferbaum, \& Sullivan, 2009) suggest some association of callosal and cognitive development across the lifespan. These studies support the notion that the observed structural changes in the corpus callosum are not only a marker, but also contributor to the development of cognitive abilities across the lifespan.

Studies addressing the development of the corpus callosum usually consider parallel changes in overall brain size as a potential confounding factor. The size of the corpus callosum (e.g., 
measured as midsagittal surface area) is significantly related to brain size (Jancke, Staiger, Schlaug, Huang, \& Steinmetz, 1997) so that changes in forebrain volume - which can be found across the entire the lifespan (e.g., gray matter: (Tamnes et al., 2013), white matter: (Westlye et al., 2010) will likely also affect callosal measures. However, while the need to account for brain size differences is widely acknowledged in the literature, the method of doing so, varies between studies. Most authors consider brain size a confounding variable and thus correct for it by including brain-size measures (e.g., total intracranial or forebrain volume) as covariate of non-interest into the statistical analysis. Variance in corpus callosum size that can be explained by brain size differences is accordingly removed from the analysis. While this approach appears straight-forward, we here argue that important additional information can be obtained by analysing the relationship of callosal to brain size rather than removing the effect of brain size from the analysis. That is, by calculating ratios of the two variables the proportionality of the size of corpus callosum in differently sized brains can be evaluated (Holloway \& de Lacoste, 1986; Smith, 2005). Such ratios, often incorrectly dismissed (cf. (Smith, 2005), for an excellent discussion), are especially appealing when approaching the corpus callosum from a lifespan perspective, as any growth or decline of the ratio indicate over-proportional changes in interhemispheric connectivity in the a studied time period, potentially marking changes in the way the two hemispheres interact. Thus, it appears somewhat surprising that ratios have been rarely used in developmental or aging research. The few studies that do, however, indeed indicate an over-proportional increase in relative corpus callosum size during development (Rauch \& Jinkins, 1994) and an over-proportional decline in older age (D. Salat et al., 1997; Skumlien, Sederevicius, Fjell, Walhovd, \& Westerhausen, 2018). However, these previous studies rely on comparatively small, cross-sectional samples of restricted age range, and functional significance of the over-proportional changes on cognition are understudied.

The aim of the present study, is to investigate the proportionality of callosal development issue longitudinally across the lifespan (sample age: 4-93 years, 1867 datasets), examining cognitive consequences of these changes.. To account for potential regional differences within the corpus callosum, we measured thickness of the corpus callosum at 60 segments along the structure and used the ratio of segmental thickness by adjusted forebrain volume (henceforth referred to as relative corpus callosum thickness) as the dependent variable of all analyses. As non-linear associations of age and changes in relative corpus callosum thickness were expected (Rauch \& Jinkins, 1994; D. Salat et al., 1997; Skumlien et al., 2018), we employed generalized additive mixed models (GAMM) to fit lifespan trajectories for each segment, which then were clustered to form callosal subsections of comparable lifespan development. Finally, mediation analyses was used to determine whether changes in relative callosal thickness in these clusters contribute to observed 
age-related trajectories in higher cognition (i.e., Matrix reasoning and Vocabulary subtest of the Wechsler scale; (Wechsler, 1999)) across the lifespan.

\section{Material and methods}

\subsection{Participants}

The final sample consisted of a mixed longitudinal and cross-sectional sample of 1867 observations from 1014 (608 female) unique participants, covering an age range from 4 to 93 years (mean of 33.8 years, standard deviation, $\mathrm{sd}=24.4$ years). The data was drawn from three imaging studies coordinated by the Lifespan Changes in Brain and Cognition (LCBC) research center, University of Oslo, Norway (www.oslobrains.no). That is, the data included all datasets of the first, second, and third wave of the "Norwegian Mother and Child Cohort Neurocognitive" study (NeuroCogMoBa; e.g., (Krogsrud et al., 2016)), the first and second wave of the Neurocognitive Development study (NeuroCogDev; e.g., (Tamnes et al., 2010)), and of the first, second, third, and fourth wave of the "Cognition and Plasticity through the Lifespan" project (Fjell et al., 2008). All original studies excluded participants with history of neurological conditions, as well as participants with any counter-indication for magnetic resonance imaging or claustrophobia. Additionally, the sample was restricted to right-handed participants only (assessed via self-report). All studies received ethical clearance by the Regional Ethics Committee for medical research (REK-Vest). All participants included in the present analysis additionally gave informed consent to data integration across these studies.

\subsection{MRI data acquisition}

The data acquisition was conducted on two different scanners located in the Oslo University Hospital: a 1.5 Tesla Avanto and a 3 Tesla Skyra system (both Siemens Medical Solutions, Erlangen, Germany). On both platforms, comparable 3D T1-weighted magnetization prepared rapid gradient echo (MPRAGE) sequence were used. On the Avanto system, using a 12 channel head coil, the sequence (repetition time, $\mathrm{TR}=2400 \mathrm{~ms}$; echo time, $\mathrm{TE}=3.61 \mathrm{~ms}$; inversion time, $\mathrm{TI}=$ $1000 \mathrm{~ms}$; flip angle $=8$ degrees $)$ covered 160 sagittal slices $(1.2 \mathrm{~mm}$ thickness, $192 \times 192 \mathrm{scan}$ matrix, field of view of $240 \times 240 \mathrm{~mm}^{2}$ ), yielding an image resolution of $1.25 \times 1.25 \times 1.20 \mathrm{~mm}^{3}$. On the Skyra system, a 24-channel head coil was used, and using a turbo field echo pulse sequence $(\mathrm{TR} / \mathrm{TE} / \mathrm{TI}=2300 \mathrm{~ms} / 2.98 \mathrm{~ms} / 850 \mathrm{~ms}$; flip angle $=8$ degrees $)$ with 176 sagittal slices $(256 \times 256$ scan matrix) and an isometric resolution of $1 \mathrm{~mm}^{3}$. For most children under 9 years, integrated parallel acquisition techniques (iPAT) were used, acquiring multiple T1-weighted scans within a short scan time, to discard scans with residual movement and to average the scans with sufficient 
quality. A total of 1408 datasets (75.4\% of all) were collected with the Avanto and 459 datasets $(24.6 \%)$ on the Skyra system.

\subsection{Measurement of the corpus callosum}

Callosal thickness was determined on the midsagittal surface of the white-matter segmentation of the T1-weighted images in native space. Thickness was used as it is less dependent on individual differences in the curvature of the corpus callosum and offers higher spatial specificity than the widely applied geometrical subdivision solutions (Luders, Narr, Zaidel, Thompson, \& Toga, 2006). The extraction of thickness measures consisted of two main processing steps: a semi-automated segmentation of the corpus callosum and outline-based thickness determination (see also (Westerhausen et al., 2016; Westerhausen et al., 2018)).

The segmentation started with a rigid-body coregistration (i.e., preserving size and shape of the corpus callosum) of the raw images of each individual image to a T1-template using SPM12 routines (Statistical Parametric Mapping, Wellcome Department of Cognitive Neurology, London, UK) with the aim to guarantee a non-tilted midsagittal plane, located between the cerebral hemisphere in the longitudinal fissure. Then, the resulting reoriented images were segmented using standard SPM12 segmentation routines, whereby only the white matter maps in native space were used for further analyses. That is, the midsagittal slice was identified on these images and a preliminary segmentation of the corpus callosum was obtained. Based on routines written in Matlab (MathWorks Inc. Natick, MA, USA) the callosal segmentation for each individual was then visually inspected and manually corrected where necessary. A frequent reason for manual correction was that voxels belonging to the fornix were fused with the corpus callosum. Afterwards, the tip of the rostrum (posterior-most voxel of the in-bend anterior half) and the base of the splenium (ventralmost voxel in the posterior half) were manually identified, and the total callosal mask was rotated so that the imagined line between rostrum tip and splenium base was horizontally oriented.

For quality control, the above manual steps were performed by two independent raters (V.M.D., R.W.) on a random sample of 143 datasets for each of which both Avanto (1.5T) and Skyra (3T) images were available. Inter-rater reliability was then calculated for midsagittal surface area (i.e., the total number of voxels in the corpus callosum mask), yielding intra-class correlations of $\mathrm{r}_{\mathrm{icc}}=.86$ and .96 (calculated as two-way random effects, considering the single measure and absolute agreement) for Avanto and Skyra data, respectively.

For thickness determination, the outline of callosal midsagittal surface was determined whereby the tip of the rostrum and the base of the splenium served as divider between a ventral and dorsal part of the outline. To obtain a reference line for the thickness measurement, a midline between ventral and dorsal outline was determined based on 100 support points spaced 
equidistantly on the two outlines. Regional thickness was defined as the distance between the ventral and dorsal outline orthogonal to this reference midline. For the actual measurement, the midline was resampled into 60 equidistant points, which then served as measurement points. The number of 60 measurement points represents a compromise between the previously used 29 to 100 points (Clarke et al., 1989; Luders et al., 2006) as it both provides a sufficiently high density of sampling points to capture the structure of corpus callosum and does not inflate the number of statistical tests excessively (Westerhausen et al., 2016).

In a final preprocessing step, as the present analysis aims to examine the proportionality of the corpus callosum relative to brain size across the lifespan, the raw 60 segment thickness estimates were related to the forebrain volume (see section 2.3). As illustrated by Smith (2005), the ratio of two features of a geometrical object (e.g., like the radius and volume of a sphere) only is invariant to the size of the object, if both features (i.e., numerator and denominator of the ratio) are expressed in the same unit. As callosal thickness is a measure of distance and forebrain size represents a volume, we transformed the forebrain volume by raising it to the power of $1 / 3$ (i.e, $\mathrm{FBV}^{0.333}$ ) before the division. In other words, forebrain volume was converted to a unit that remains proportional to callosal thickness if the brain maintains geometric similarity with changing size. Thus, the ratio is expected to be stable across the lifespan if corpus callosum thickness changes proportional to forebrain volume (with respect to the observed features). However, any positive or negative deviation from a stable ratio indicates over-proportional growth and decline of the corpus callosum, respectively.

For a supplementary analysis, following the same approach, we also calculated the ratio of segmental thickness to white-matter volume (i.e., $\mathrm{WMV}^{0.333}$ ).

\subsection{Measurement of forebrain and white-matter volume}

Forebrain volume was assessed from the T1 images using the supratentorial volume provided by the segmentation routines of Freesurfer (version 6.1, (Fischl, Sereno, \& Dale, 1999)). The supratentorial volume represents an optimal measure, as it includes all brain compartments which have connections through the corpus callosum, and excludes those brain structures (i.e., cerebellum and brain stem) which do not exhibit callosal axons. As indicated above, we additionally extracted the total white-matter volume of both cerebral hemisphere for a supplementary analysis.

\subsection{Fitting and clustering of callosal lifespan trajectories}

The lifespan trajectories of relative corpus callosum thickness were fitted by using GAMM ("mgcv" package, v1.8-28, (Wood, 2017), in in R 3.6.1) for each of the 60 segments. For each segment, relative thickness served as dependent variable and the participants' age as the predictor of interest. 
Age was smoothed using cubic regression splines with the basis dimension set to 10 knots. The participants' Sex and Scanner Type were added as covariates of non-interest as part of the fixedeffects model, while a participant identifier was included as random effects (intercept). The model fitting was done using restricted maximum likelihood (REML) estimations. Effect size of the Age effect was expressed as proportion of explained variance $\left(\omega^{2}\right)$.

In a next step, we calculated the first derivatives of the fitted trajectories, representing the slope (i.e., the change) of the lifespan trajectories at a given age, obtained a dissimiliarity matrix, and submitted these to a cluster analysis. Clustering was performed using the Partitioning Around Medoids (PAM) algorithm as implemented in the "cluster" R package (v2.1, (Maechler, 2019), in R 3.6.1). Cluster solutions between 2 and 10 clusters were estimated, and the mean silhouette widths was used to determine the optimal cluster solution. The resulting cluster solution was additionally inspected for their topographical organization, that is, clusters which contained thickness segments at different, non-adjacent locations where split up into sub clusters for spatial consistency. Finally, for each resulting cluster mean thickness across all segments assigned to that cluster was calculated for subsequent analysis steps.

For descriptive purposes, the lifespan trajectories of the obtained cluster mean values were also submitted to GAMM modelling using the same model as for the analysis of the original segments. Then, the derivate of the such fitted cluster trajectories were used to determine the estimated age marking the end of growth $(\mathrm{EoG})$ and the beginning of decline $(\mathrm{BoD})$, defined as the last estimate of the slope above and the first below zero, respectively. Confidence intervals (95\%) around the derivative trajectory served to determine where the slope deviated from zero.

Of note, for matter of completeness, all the above analysis steps were also conducted for the ratio of segmental thickness and $\mathrm{WMV}^{0.333}$. See supplement Fig. S1 and Table S3.

\section{Cognitive assessment}

For a subsample of 479 unique participants and a total of 1117 observations (i.e., 320 assessed at two time points and 159 at three time points) test scores for the Vocabulary and Matrix Reasoning were available in the LCBC data base, which were collected with age-appropriate Norwegian versions of the Wechsler intelligence test battery. That is, subtests of the Wechsler Preschool and Primary Scale of Intelligence (WPPSI-III, (Wechsler, 2002)) were used to assess children below the age of 6.5 years $(n=131$ observations), and of the Wechsler Abbreviated Scale of Intelligence test (WASI, (Wechsler, 1999)) for all participants above this age $(\mathrm{n}=986)$. The vocabulary subtest measures word knowledge and crystallised cognitive abilities. Matrix Reasoning, testing visualspatial abilities and perceptual organisation, assesses non-verbal fluid (performance) cognitive abilities. For the present analysis, raw test scores were used (rather than age-standardised, norm 
deviation scores) allowing to assess the development of absolute cognitive changes across the lifespan (Neisser, 1998; Westerhausen et al., 2018). Of note, the present subsample contains all participants of the total sample for whom cognitive data for at least at two time points was available. This restriction was necessary as it allows calculating change scores on individual level (discussed below). Of note, all cognitive testing was done within a month around the MRI acquisition.

To confirm the premise of the present analyses, we also fitted the lifespan trajectories of the two cognitive variables using GAMM. The models including Age (smoothed using cubic regression splines) in the fixed-effect part of the model and a participant identifier variable as random effects (intercept). Sex and test version (WPPSI-II vs. WASI) was included as covariates of non-interest. As shown in Fig. 1, the lifespan trajectory of Matrix Reasoning describes an inverted U-shaped association, with a strong increase in performance in the first two decades of life and peaking in the early 20 s, followed by a slow decline, which appears accelerated beyond the age of $60(F=134.0$, with effective degrees of freedom, $e d f$, of 8.13, yielding a $p<.0001$, explained variance, $\omega^{2}=.49$ ). The trajectory of the Vocabulary test score, was characterized by a steep increase until about an age of 20 , followed by slow continuing increase in performance until the age of 40 years $(F=620.6$, edf $\left.=7.87, \mathrm{p}<.0001 \omega^{2}=.81\right)$. Taken together, both cognitive variables show age trajectories that would be predicted from previous literature (e.g., (Craik \& Bialystok, 2006).
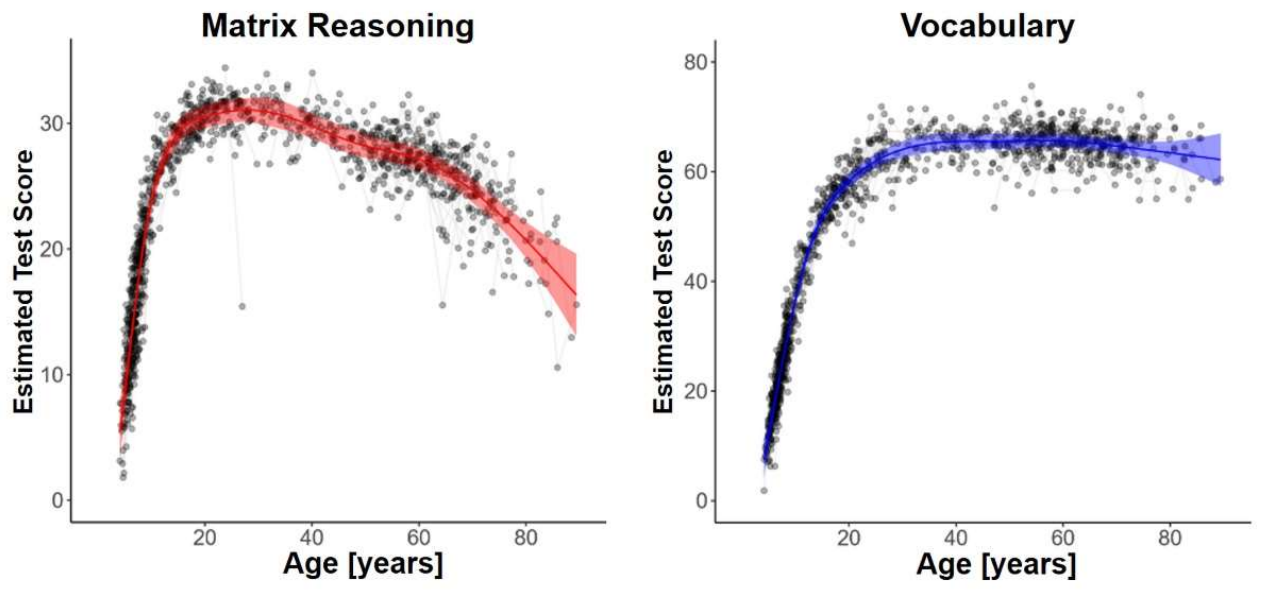

Figure 1. Fitted lifespan trajectories (solid line; shaded area 95\% confidence bands) for Matrix Reasoning and Vocabulary test scores using generalized additive mixed modelling (GAMM). Considering Matrix Reasoning, the effective degrees of freedom, edf, was 8.13 with Age explaining $49 \%$ of the variance in the test score. Considering the Vocabulary test score, edf $=7.87$ and $81 \%$ variance were explained by Age. See text for more details regarding the GAMM analysis. 


\subsection{Mediation analysis}

The second research question is concerned with the relation of callosal and cognitive development across the lifespan, which was here formulated as mediation hypothesis (as illustrated in Fig. 2). That is, are age-related (predictor, $\mathrm{X}$ ) changes in cognitive abilities (dependent variable, $\mathrm{Y}$ ), substantially mediated by changes corpus callosum measures (mediator, $\mathrm{M}$ )? To test this hypothesis, we calculated change scores for each callosal cluster and each cognitive test separately. The change score mediation was preferred over raw scores, in order to reduce the complexity of the mediation analysis. That is, for the presentrelatively short inter-testing time intervals (for the 320 participants with two time points the mean interval was 2.54 years with a standard deviation of 1.46 years; for the participants with 3 time points it was $7.82 \pm 0.81$ years) linear associations were assumed so that non-linear associations had not to be considered during the mediation analysis. Change scores were obtained as follows. Considering cognitive and corpus callosum variables, linear regressions were fitted for each participant (i.e., across 2 and 3 individual time points) and the obtained slopes were extracted as individual change scores, representing estimated annual change in callosal and cognitive measures, respectively. The change in age was calculated as difference in age between first and last testing.

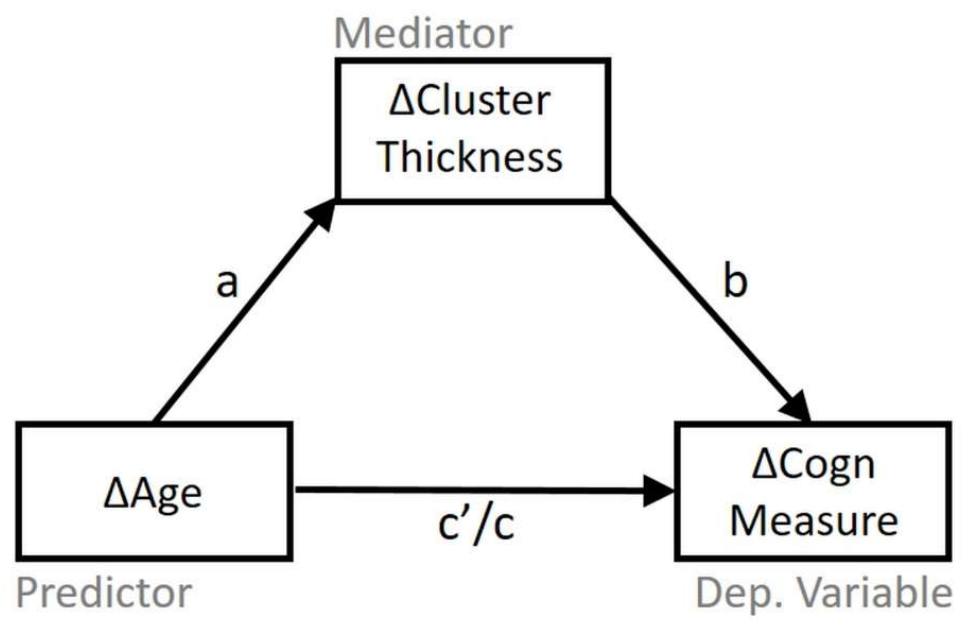

Figure 2. Path diagram for the change mediation analysis model. Parameter $a$ is the unstandardized regression weight for $\Delta \mathrm{Age}$ for the prediction of changes in mean relative thickness within a cluster ( $\Delta$ Cluster Thickness, i.e. the mediator variable). Parameter $b$ is the regression weight for the mediator predicting the change in the dependent variable ( $\Delta$ Cogn Measure). Parameter $c$ ' represents the regression weight of the direct effect of $\Delta$ Age on the change in cognition, while parameter $c$ represents the same prediction without considering the mediator variable. The indirect effect (mediation) is estimated as combined effect $a^{*} b$. Changes in mean relative thickness as obtained from all clusters served as mediator(see section 3.1). Dependent variables were changes in Matrix Reasoning or Vocabulary test score. 
Furthermore, mediation analysis is only considered meaningful if the pairwise associations of the predictor and mediator, and mediator and dependent variable are established (Hayes, 2009). Thus, a formal mediation analysis was only considered for those clusters where both associations were significant $(\mathrm{p}<.05)$. This was tested using pairwise linear regressions. The mediation of change analysis was then set up as illustrated in Fig. 2. The effect of interest was the indirect (or mediation) effect, constituted by the multiplication of the coefficients of regression coefficients of pathways $a$ and $b$, and henceforth expressed as $a b$. For interpretation, the total effect of the model, that is, the combined effect of change in age and the mediator on the cognitive change score, was also calculated $(c)$. Effect size of all effects were expressed as $\omega^{2}$. Dividing the effect size of the indirect effect by the effect size of the total effect, allows to estimate the proportion of variance of the age effect on cognition is mediated via callosal changes.

The change mediation analysis was conducted using the Lavaan package (version 0.6-4, (Rosseel, 2012), in in R 3.6.1), using bootstrapping (5000 iterations) to estimate parameter confidence intervals and p-values. P-values of the conducted mediation analyses were corrected to achieve a false discovery rate (FDR) of 5\%.

\section{Results}

\subsection{Lifespan trajectories and clustering}

Significant age-trajectory fits were established in all segments of the corpus callosum (see supplementary Table S1). However, substantial variability between the segments was found both in complexity (showing edf between 1.00 and 6.73) as well as in the proportion of explained variance by the Age smooth term (between $\omega^{2}=.03$ and .20 ), together supporting the idea of developmental differences between callosal segments and warranting the following cluster analysis.

The PAM clustering revealed a five-cluster solution for these trajectories, based on the local maximum in the silhouette coefficient (Fig. 3A) - resulting in clusters that are topographically organized along the corpus callosum (Fig. 3B). Fig. 3C shows trajectories of the estimated mean and derivatives for the initial cluster solution.

The first cluster encompassed 5 segments located in the rostrum and ventral genu of the corpus callosum. The segments falling into this cluster showed low complexity (indicated by a mean edf $=2.25, \mathrm{sd}=0.86$ of the GAMM fitting), which can be best summarized as a linear decline across the lifespan (see Fig. 4). The second cluster contained segments in the genu and the dorsal splenium of the corpus callosum and was accordingly split into two sub-clusters to reflect this spatial separation (see green segments Fig. 3B). The anterior cluster (2a) consisted of 7 segments 
(mean edf $=4.90 \pm 0.21)$ and the posterior $(2 p)$ of 3 segments (mean edf $=5.54 \pm 0.42)$. The trajectories of both clusters are characterized by a slow but over-proportional increase of the subsection with an apparent peak around the age of 24 and 17 years (end of growth) for cluster 2a and $2 p$, respectively, followed by a plateau, turning into a slow decline at the age of 43 and 51 years. The third cluster included 33 segments located in posterior genu, truncus, and isthmus. The cluster also included two single segments located distant from the main cluster, one in the genu and one in the splenium (cf. Fig. 3B), which were removed from further analyses steps as they represented spatial outliers. Considering the remaining 31 segments the cluster showed a mean complexity of edf $=4.67 \pm 0.27$. The trajectory analysis revealed an increase of the mean ratio in this cluster until about the age of 22 years, followed by a plateau until the age of 41 years, and a steady decline into older age. The fourth cluster encompassed the middle splenium ( 8 segments, average edf $=6.34 \pm 0.08$ ). It is characterized by an increase in relative thickness until the age of 23 , followed by a slow decline which, however, only is significant from the age of 53 years onwards. The final cluster, cluster 5, consisted of four segments and is located in the ventral splenium. This cluster showed the highest complexity with edf $=6.53 \pm 0.16$. The mean trajectory showed a clear increase in relative thickness until the age of 23 years, leading to a period of stable ratio (see Fig. 4), which only at the age of 59 years turned into a significant decline.

A supplementary analysis conducted for the ratio of segmental thickness and $\mathrm{WMV}^{0.333}$, suggested a 4-cluster solution (c.f. Fig. S1 and Table S3). Compared to the above analysis, the splenium consisted of one rather than two clusters while otherwise showing a largely consistent spatial distribution. 
A

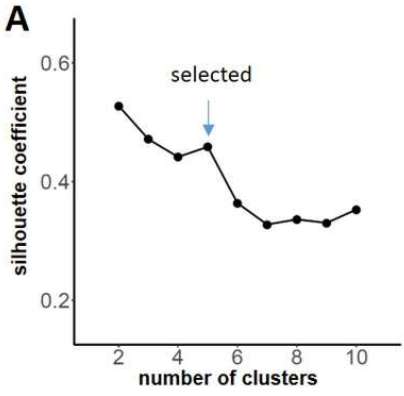

B

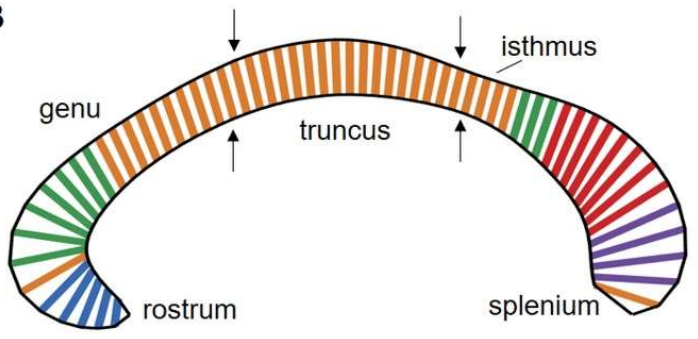

C

cluster 1

cluster 2

cluster 3

cluster 4

cluster 5

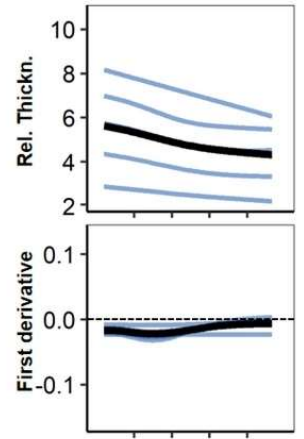

20406080
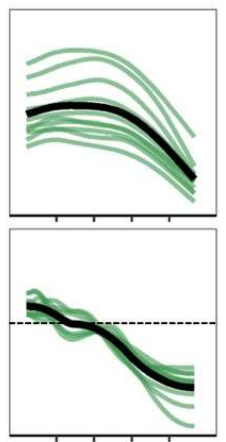

20406080

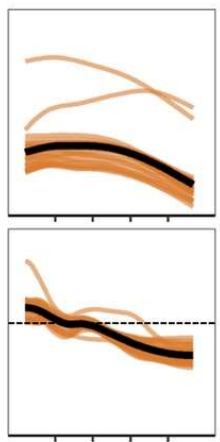

20406080

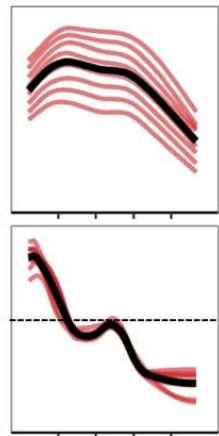

20406080

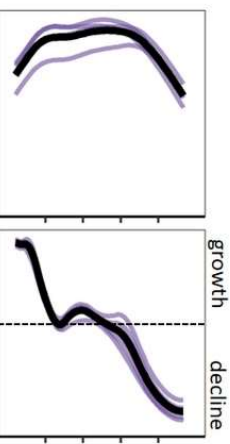

$204060 \quad 80$

Figure 3. Initial PAM clustering of the callosal segments based on the first derivative of the lifespan trajectories. Dependent variable was callosal thickness relative to total forebrain volume. Panel A shows the silhouette coefficient for cluster solutions between 2 to 10 clusters. Here, a 5-cluster solution was selected as it showed a clear peak in the silhouette coefficient and yielded a consistent cluster topography as seen in Panel B. Panel B depicts the segment-wise color-coded topography of the clusters within the corpus callosum (left: anterior) for the 5 cluster solution. Black arrows indicate the location which divides the corpus callosum into thirds relative to the anterior-posterior length. This marks the subdivision into genu, truncus, posterior third according to the widely applied geometric subdivision approach suggested by Witelson (1989). Panel C presents the lifespan trajectories of mean relative thickness (top row) and their first derivatives (bottom row) for each segment (different lines) per cluster. Bold black lines in each graph represents the mean trajectory. Of note, positive values in the derivative plots indicate an over-proportional growth of relative thickness while negative values indicate an over-proportional decline. 


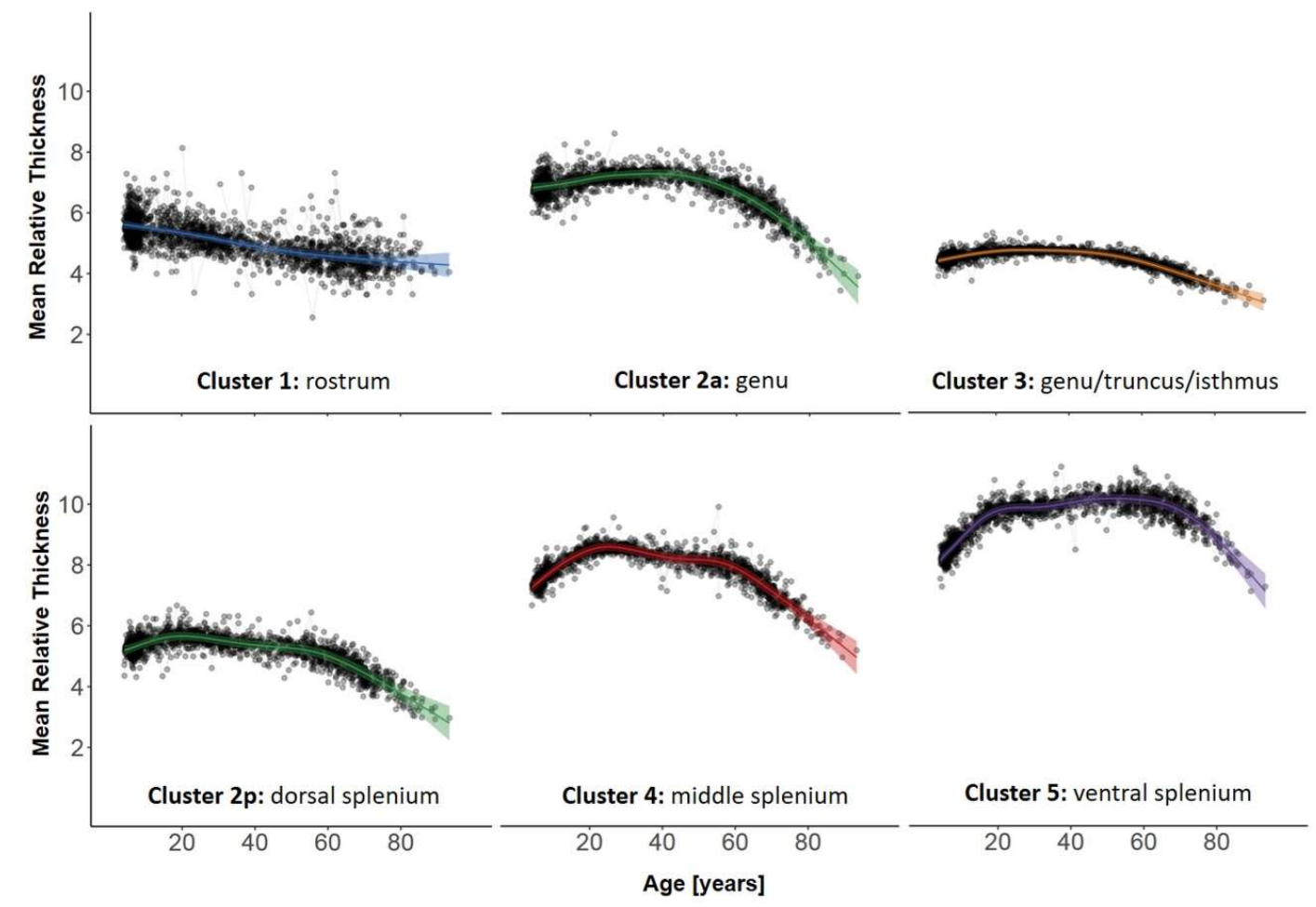

Figure 4. Spaghetti plots and fitted lifespan trajectories of relative callosal thickness (solid line; shaded area 95\% confidence bands) for the final cluster solution. Colors correspond to the color-coding used in Fig 3B/C. For details see section 3.1 .

\subsection{Corpus callosum and cognitive abilities}

For six of the potentially twelve combinations of callosal clusters (six clusters) and cognition (two variables), the pairwise associations of the change in age and change in relative callosal thickness, and relative callosal thickness and cognitive changes were significant at the same time (see Table S2). Thus, the mediation analysis was considered only for these six combinations. As can be seen in Table 1, the mediation effect was significant in two of these analyses. Firstly, the change in relative thickness in cluster 4 significantly mediated the effect of Age changes on the Vocabulary test (indirect effect: $a b=-0.05$, s.e. $=0.02, \mathrm{Z}=-2.5, p_{\text {corr }}=.049, \mathrm{CI} 95 \%=[-0.090,-0.012]$ explaining $1 \%$ of the variance. The total effect was significant $\left(c=-0.43\right.$, s.e. $\left.=0.05, Z=-8.94, p_{\text {corr }}<.001\right)$ with an effect size of $\omega^{2}=0.14$. Thus, the indirect effect explained about $7 \%$ of the total effect. Secondly, the mediation for cluster 4, was also significant considering the Matrix Reasoning ( $a b=$ 0.04 , s.e. $=0.02, Z=-2.40, p_{\text {corr }}=.049$, CI95\% $\left.=[-0.073,-0.007]\right)$, with an effect size of $\omega^{2}=.01$. The total effect was also here significant $\left(c=-0.19\right.$, s.e. $\left.=0.04, Z=-5.08, \mathrm{p}_{\mathrm{corr}}<.001\right)$, yielding an effect size of $\omega^{2}=.049$. The indirect effect thus accounted for about $20 \%$ of the total effect. For the remaining analyses the indirect effect was not significant (all $\mathrm{p}_{\text {corr }}>0.169$, all $\omega^{2}<0.004$ ), and details are provided in Table 1. 


\begin{tabular}{|c|c|c|c|c|c|c|c|c|c|c|c|}
\hline \multirow[b]{2}{*}{ Dep. Variable } & \multirow[b]{2}{*}{ Cluster } & \multicolumn{2}{|l|}{ Path $a$} & \multicolumn{2}{|l|}{ Path $b$} & \multicolumn{2}{|c|}{ Path $c^{\prime}$ (direct) } & \multicolumn{2}{|c|}{ Path $c$ (total) } & \multicolumn{2}{|c|}{ Path $a b$ (indirect) } \\
\hline & & b-value & $\mathrm{p}$ & b-value & $\mathrm{p}$ & b-value & $\mathrm{p}$ & $\mathrm{b}$-value & $\mathrm{p}$ & b-value & $\mathrm{p}$ \\
\hline Matrix Reason. & 4 & -0.019 & $<0.001^{*}$ & 2.068 & $0.012 *$ & -0.148 & $<0.001^{*}$ & -0.188 & $<0.001^{*}$ & -0.040 & $0.016^{*}$ \\
\hline \multirow[t]{3}{*}{ Vocabulary } & 3 & -0.008 & $<0.001^{*}$ & 2.56 & 0.131 & -0.406 & $<0.001^{*}$ & -0.427 & $<0.001^{*}$ & -0.021 & 0.177 \\
\hline & 4 & -0.019 & $<0.001^{*}$ & 2.594 & $0.008^{*}$ & -0.377 & $<0.001^{*}$ & -0.427 & $<0.001^{*}$ & -0.05 & $0.012^{*}$ \\
\hline & 5 & -0.018 & $<0.001^{*}$ & 0.766 & 0.36 & -0.413 & $<0.001^{*}$ & -0.427 & $<0.001^{*}$ & -0.014 & 0.384 \\
\hline
\end{tabular}

FDR correction across the six mediation analyses (see text)

\section{Discussion}

A plethora of studies has established that the corpus callosum undergoes substantial developmental changes across the lifespan that follow an inverted-u-shaped trajectory. Histological, morphometric, and diffusion imaging studies suggest that structural hemispheric connectivity is built up well into the third decade of life (Hasan et al., 2009; Lebel et al., 2010; Prendergast et al., 2015; Pujol et al., 1993; Yeatman et al., 2014), and that it starts to decline around the age of 60 years (Doraiswamy et al., 1991; Hasan, Kamali, et al., 2008; Hou \& Pakkenberg, 2012; D. Salat et al., 1997; Sullivan et al., 2010). The present findings utilizing relative callosal thickness measures, while in line with this general pattern, provide a series of important specifications to the literature.

Firstly, our findings demonstrate that the lifespan changes in the size of the corpus callosum are not proportional to changes forebrain size. Extending the findings of a couple of earlier studies (Rauch \& Jinkins, 1994; D. Salat et al., 1997) to a lifespan perspective, we here show that the growth during childhood, adolescence, and young adulthood is over-proportional in most segments of the corpus callosum, as the ratio of callosal thickness to forebrain volume increases in this period of life. That is, the end of growth is not reached before the end of teenage years or the first half of the twenties, depending on the subsection. Thus, comparable to findings in studies using brain-size measures as covariate (Prendergast et al., 2015) or diffusion parameters (Hasan, Ewing-Cobbs, Kramer, Fletcher, \& Narayana, 2008; Hasan et al., 2009; Lebel, Walker, Leemans, Phillips, \& Beaulieu, 2008), also when considering relative callosal thickness measures, an increase in structural connectivity into the third decade of life can be observed. Increased callosal thickness during development likely reflects an increase in axon myelination rather than the formation of new axons, as the number of axons is known to decreases rather than increase after birth (Clarke et al., 1989; Innocenti \& Price, 2005; LaMantia \& Rakic, 1990). On the other hand, in older age the ratio decreases substantially, indicating an over-proportional decline of relative hemispheric connectivity. Here the changes are likely driven by alterations of axon myelination as well as a 
decrease in the number of callosal axons as has been demonstrated in histological studies in human and other primates (Aboitiz, Rodriguez, Olivares, \& Zaidel, 1996; Hou \& Pakkenberg, 2012; Peters \& Sethares, 2002).

Beyond the general pattern, we also observed that the aging trajectories differ between callosal sections. Cluster 1, encompassing the rostrum and parts of the ventral genu, is characterized by a slow decline across the covered age range, while all other clusters show a significant increase in relative callosal thickness into young adulthood. However, the trajectories of the two most posterior clusters (i.e, clusters 4 and 5 located in the splenium) also differ from the more anterior clusters (i.e, clusters $2 \mathrm{a}, 2 \mathrm{~b}$, and 3, covering genu, truncus, and isthmus) in two important ways. The growth of relative thickness of the posterior corpus callosum is accentuated during development (see e.g., derivatives in Fig 3C) and its decline in old age is delayed by about 10 years. This accentuation of posterior callosal development appears particularly salient when analyzing relative thickness using forebrain WMV rather than FBV as denominator in the ratio (see analysis in supplement, Fig. S1). Here only the posterior cluster (encompassing the entire splenium) showed significant developmental growth in relative thickness in the studied age period. Both observations are in principle in line with previous studies using raw measures of the corpus callosum or using brain size as covariate. That is, stronger posterior as compared to anterior callosal development during childhood and adolescence (Giedd et al., 1999; Luders, Thompson, et al., 2010; Rajapakse et al., 1996; Thompson et al., 2000; Westerhausen et al., 2016) as well as earlier decline in anterior as compared to posterior sections in older age have been reported (e.g., (Salami, Eriksson, Nilsson, \& Nyberg, 2012; D. H. Salat et al., 2005; Sullivan, Adalsteinsson, \& Pfefferbaum, 2006), or for a review see (Madden, Bennett, \& Song, 2009)). The posterior section of the corpus callosum, in particular the splenium, is known to house axons connecting parietal, temporal, and occipital regions (Schmahmann \& Pandya, 2006), and is thus relevant for perception-related processes, such as sensory integration between the hemispheres (e.g., (E. Genc et al., 2011; Westerhausen et al., 2009) or exogenous attentional processes (e.g., (Bozzali et al., 2012; Tomaiuolo et al., 2010)). Anterior sections, like the genu and truncus, interconnect prefrontal cortices (Schmahmann \& Pandya, 2006) and are accordingly relevant in higher cognition, including language production or semantic processing (Putnam, Wig, Grafton, Kelley, \& Gazzaniga, 2008; Thiel et al., 2006). Thus, different callosal sections are embedded in different functional interhemispheric networks and the here observed differences in trajectories likely reflect the development of these network. For example, it can be speculated that the splenium's role in basic perception processes, rather than in higher cognition, protects it longer from detectable decline in older age (Madden et al., 2009; Scally, Burke, Bunce, \& Delvenne, 2018). On the other hand, histological analyses of the corpus callosum suggest that subsections also differ substantially in their axon composition. Predominantly 
thinner and lightly myelinated axons are found in the anterior corpus callosum, while thicker and stronger myelinated axons are most prevalent in truncus and splenium (e.g., (Aboitiz, Scheibel, Fisher, \& Zaidel, 1992; Riise \& Pakkenberg, 2011), see also (Bjornholm et al., 2017)). Thus, accentuated posterior growth during childhood and adolescence might reflect an ongoing optimization of interhemispheric transfer time by increasing axon myelination, which is taking place to a lesser extent in the genu section where the axons are small or unmyelinated, or is completed earlier in the truncus regions connecting motor or sensorimotor regions.

While the exact cellular processes driving the observed lifespan trajectories cannot be determined in the present study, it can be argued that segments showing comparable trajectories also belong to related interhemispheric brain networks. Thus, shared lifespan trajectories can also be seen as a data-driven approach to define subsections within the corpus callosum. As indicated above, the corpus callosum viewed on a midsagittal cross-section is topographically organized and different subsections are part of different inter-hemispheric cortical networks (Schmahmann \& Pandya, 2006). The present clustering solution suggests subdividing the corpus callosum into five subsections, based on their distinct developmental trajectories across the lifespan. However, the resulting subdivision differs partially from previously suggested subdivisions. For example, the best known and widely employed Witelson approach (Witelson, 1989) suggests dividing the corpus callosum into thirds along its rostro-caudal extent of the midsagittal structure, to define genu, truncus, and splenium, respectively (cf. arrows in Fig. 3B). In contrast to our approach, the subdivision rules of the Witelson or other geometrical methods, is more or less arbitrary, and the resulting parcellation will likely not match the topography of the callosal connections.

Alternatively, it has been suggested to utilize diffusion MRI based tractography results to inform callosal subdivision, that is, dividing the corpus callosum according to the origin of the reconstructed fiber pathways (e.g., (Hofer \& Frahm, 2006). While this approach appears promising, most tractography studies still suffer from restrictions to which fiber pathways actually can be reconstructed using current imaging sequences and tracking algorithms. This is particular salient when analyzing the corpus callosum, as the to-date suggested tractography-based subdivisions are mainly based on connections originating from the medial brain surface, missing most of the lateral connections (e.g., (Hasan et al., 2009; Lebel et al., 2010; Sullivan et al., 2010)), which, however, are known to exist from histological and lesion studies (Schmahmann \& Pandya, 2006). Thus, defining subsections based on the trajectories of development across the lifespan might be seen as a valid alternative to identify subsections within the midsagittal corpus callosum.

The third finding is that change in relative callosal thickness, specifically in the middle splenium (cluster 4), mediates age-related changes in both Matrix Reasoning and Vocabulary test. Parameter $b$ of the mediation analysis is positive (see Table1), indicating that an increase in relative 
callosal thickness is associated with an increase in cognitive performance, and a decline is associated with a drop in performance. Comparable positive associations of callosal measures and measures of intelligence, have been reported in a series of cross-sectional studies, demonstrating a correlation of intelligence test performance with corpus callosum macro-anatomy or factional anisotropy in developing (e.g., (Westerhausen et al., 2018)) or adult samples (Chiang et al., 2009; Dunst, Benedek, Koschutnig, Jauk, \& Neubauer, 2014; Luders et al., 2007; Tang et al., 2010). However, the sign of the indirect effect $a b$ is negative, as parameter $a$ is negative. Parameter $a$ represents the association of changes in age and changes in relative callosal thickness, and is negative as a direct consequence of the data collection schemes of the original studies (see (Fjell et al., 2008; Krogsrud et al., 2016; Tamnes et al., 2010)). Short retest intervals were more frequent in the young sample in which changes are more rapid (see Fig. 3) than in the older, slow changing sample, resulting in the observed negative association. Likewise, the direct effect, that is, the relationship between change in age and change in cognition was found to be negative due to the different sampling in young and old individuals (see parameters $c$ or $c^{\prime}$ ). Importantly, this effect of sampling does not affect the interpretation of the significant mediation effect, as it still shows that changes in relative callosal thickness partially mediate the effect that aging has on changes in cognition. Which is, following parameter $b$, that an over-proportional decline of cluster thickness in a given time interval, results in a more accentuated drop in performance. Reversely, an overproportional callosal growth goes along with a more accentuated increase in performance.

One might argue that the found mediation effects are, with about $1 \%$ explained variance, rather small. On the other hand, at least in case of the Matrix Reasoning score where the total effect is $5 \%$ explained variance, the changes in relative corpus callosum thickness explain about $20 \%$ of the total effect of aging on cognitive changes (paramter $c$ ), suggesting the effect to be nonnegligible. In this, the callosal contribution to changes in Matrix Reasoning is also substantially stronger than the $7 \%$ observed for the vocabulary score. This special relevance for the Matrix Reasoning test, is in line with a recent meta-analysis suggesting that callostomy affects performance but not verbal IQ in patients who have an average performance levels before the surgery (Westerhausen \& Karud, 2018). Furthermore, it has to be considered that the Vocabulary or Matrix Reasoning test, might not be the best instruments assessing callosal functioning. In the past, structure-function associations in the corpus callosum have been mainly reported for paradigms assessing early perceptual processes, namely when information initially present in different sensory half fields has to be integrated across the hemispheres to form a representation in working memory (E. Genc et al., 2011; Steinmann et al., 2018; Westerhausen et al., 2009) often emphasising processing speed and rapid stimulus-response mapping (e.g., (Huster, Westerhausen, \& Herrmann, 2011; Schulte, Müller-Oehring, Salo, Pfefferbaum, \& Sullivan, 2006; Westerhausen et al., 2006; 
Whitford et al., 2011). Thus, it is conceivable that IQ test battery subtests requiring fast stimulus processing (e.g., Symbol search test of the WASI test battery) would reveal more pronounced effects of changes in relative callosal thickness (Kennedy \& Raz, 2009; Salami et al., 2012). This notion is supported by studies showing that general information-processing speed significantly mediates the association of white-matter and intelligence (Penke et al., 2012). Processing speed was, however, not assessed in the present sample so that it remains for future studies to test this hypothesis.

Another limitation of the present study is that the sample does not include participants under the age of 4 years. Previous studies suggest a rapid postnatal of the corpus callosum midsagittal area or thickness (Clarke et al., 1989; Rakic \& Yakovlev, 1968; Tanaka-Arakawa et al., 2015), doubling in size in the first year alone (Vannucci et al., 2017). Thus, neither the present clustering solution of the callosal segments nor the analysis of the cognitive relevance of callosal changes, includes this age period of rapid changes. Future studies consequently should focus on utilizing younger samples, as to the best of the authors' knowledge the early development of relative corpus callosum size has not been studied systematically.

\section{Conclusion}

The present study revealed the trajectories of callosal development across the lifespan, emphasizing that measures of relative corpus callosum thickness provide an additional measure of hemispheric connectivity. The here demonstrated over-proportionality of callosal growth during development and callosal decline in aging, respectively, have been mostly neglected in the past, although these may, as we have shown here, have consequences for the way the brain relies on hemispheric interaction. One might further speculate that during infancy, childhood, and adolescence an increasingly strong involvement of interhemispheric integration in perception and cognition is established by increasing the relative size of the corpus callosum. In older age, this adult level of hemispheric integration is challenged by the over-proportional decline, potentially threatening normal cognition by partially "disconnecting” interhemispheric functional networks. However, future studies need to address the antecedences and consequences of over-proportional callosal growth and decline systematically by utilizing more appropriate measures of hemispheric interaction. 


\section{References}

Aboitiz, F., Rodriguez, E., Olivares, R., \& Zaidel, E. (1996). Age-related changes in fibre composition of the human corpus callosum: sex differences. Neuroreport, 7(11), 1761-1764.

Aboitiz, F., Scheibel, A. B., Fisher, R. S., \& Zaidel, E. (1992). Fiber composition of the human corpus callosum. Brain research, 598(1), 143-153. doi:http://dx.doi.org/10.1016/0006-8993(92)90178-C

Ansado, J., Collins, L., Fonov, V., Garon, M., Alexandrov, L., Karama, S., . . . Brain Development Cooperative, G. (2015). A new template to study callosal growth shows specific growth in anterior and posterior regions of the corpus callosum in early childhood. Eur J Neurosci, 42(1), 1675-1684. doi:10.1111/ejn.12869

Banich, M. T. (1998). Integration of information between the cerebral hemispheres. Current Directions in Psychological Science, 7(1), 32-37.

Bjornholm, L., Nikkinen, J., Kiviniemi, V., Nordstrom, T., Niemela, S., Drakesmith, M., . . Paus, T. (2017). Structural properties of the human corpus callosum: Multimodal assessment and sex differences. Neuroimage, 152, 108-118. doi:10.1016/j.neuroimage.2017.02.056

Bozzali, M., Mastropasqua, C., Cercignani, M., Giulietti, G., Bonni, S., Caltagirone, C., \& Koch, G. (2012). Microstructural damage of the posterior corpus callosum contributes to the clinical severity of neglect. PLoS One, 7(10), e48079. doi:10.1371/journal.pone.0048079

Chavarria, M. C., Sanchez, F. J., Chou, Y. Y., Thompson, P. M., \& Luders, E. (2014). Puberty in the corpus callosum. Neuroscience, 265, 1-8. doi:10.1016/j.neuroscience.2014.01.030

Chiang, M. C., Barysheva, M., Shattuck, D. W., Lee, A. D., Madsen, S. K., Avedissian, C., . . Thompson, P. M. (2009). Genetics of brain fiber architecture and intellectual performance. J Neurosci, 29(7), 2212-2224. doi:10.1523/JNEUROSCI.4184-08.2009

Chicoine, A. J., Proteau, L., \& Lassonde, M. (2000). Absence of interhemispheric transfer of unilateral visuomotor learning in young children and individuals with agenesis of the corpus callosum. Dev Neuropsychol, 18(1), 73-94. doi:10.1207/S15326942DN1801_5

Clarke, S., Kraftsik, R., Van der Loos, H., \& Innocenti, G. M. (1989). Forms and measures of adult and developing human corpus callosum: is there sexual dimorphism? J Comp Neurol, 280(2), 213-230. doi:10.1002/cne.902800205

Craik, F. I., \& Bialystok, E. (2006). Cognition through the lifespan: mechanisms of change. Trends Cogn Sci, 10(3), 131-138. doi:10.1016/j.tics.2006.01.007

Davis, S. W., \& Cabeza, R. (2015). Cross-hemispheric collaboration and segregation associated with task difficulty as revealed by structural and functional connectivity. $J$ Neurosci, 35(21), 8191-8200. doi:10.1523/JNEUROSCI.0464-15.2015

Doraiswamy, P. M., Figiel, G. S., Husain, M. M., McDonald, W. M., Shah, S. A., Boyko, O. B., ... Krishnan, K. R. (1991). Aging of the human corpus callosum: magnetic resonance imaging in normal volunteers. J Neuropsychiatry Clin Neurosci, 3(4), 392-397. doi:10.1176/jnp.3.4.392

Dougherty, R. F., Ben-Shachar, M., Deutsch, G. K., Hernandez, A., Fox, G. R., \& Wandell, B. A. (2007). Temporal-callosal pathway diffusivity predicts phonological skills in children. Proc Natl Acad Sci U $S$ A, 104(20), 8556-8561. doi:10.1073/pnas.0608961104

Dunst, B., Benedek, M., Koschutnig, K., Jauk, E., \& Neubauer, A. C. (2014). Sex differences in the IQ-white matter microstructure relationship: a DTI study. Brain Cogn, 91, 71-78. doi:10.1016/j.bandc.2014.08.006

Fischl, B., Sereno, M. I., \& Dale, A. M. (1999). Cortical surface-based analysis: II: inflation, flattening, and a surface-based coordinate system. Neuroimage, 9(2), 195-207.

Fjell, A. M., Westlye, L. T., Greve, D. N., Fischl, B., Benner, T., van der Kouwe, A. J., .. Walhovd, K. B. (2008). The relationship between diffusion tensor imaging and volumetry as measures of white matter properties. Neuroimage, 42(4), 1654-1668.

Fryer, S. L., Frank, L. R., Spadoni, A. D., Theilmann, R. J., Nagel, B. J., Schweinsburg, A. D., \& Tapert, S. F. (2008). Microstructural integrity of the corpus callosum linked with neuropsychological performance in adolescents. Brain Cogn, 67(2), 225-233. doi:10.1016/j.bandc.2008.01.009

Gazzaniga, M. S. (2000). Cerebral specialization and interhemispheric communication: does the corpus callosum enable the human condition? Brain, 123 ( Pt 7), 1293-1326. Retrieved from http://www.ncbi.nlm.nih.gov/pubmed/10869045

Genc, E., Bergmann, J., Singer, W., \& Kohler, A. (2011). Interhemispheric connections shape subjective experience of bistable motion. Curr Biol, 21(17), 1494-1499. doi:10.1016/j.cub.2011.08.003 
Genc, S., Malpas, C. B., Ball, G., Silk, T. J., \& Seal, M. L. (2018). Age, sex, and puberty related development of the corpus callosum: a multi-technique diffusion MRI study. Brain Struct Funct, 223(6), 2753-2765. doi:10.1007/s00429-018-1658-5

Giedd, J. N., Blumenthal, J., Jeffries, N. O., Rajapakse, J. C., Vaituzis, A. C., Liu, H., . . Castellanos, F. X. (1999). Development of the human corpus callosum during childhood and adolescence: a longitudinal MRI study. Prog Neuropsychopharmacol Biol Psychiatry, 23(4), 571-588. Retrieved from http://www.ncbi.nlm.nih.gov/pubmed/10390717

Glickstein, M., \& Berlucchi, G. (2008). Classical disconnection studies of the corpus callosum. Cortex, 44(8), 914-927. doi:10.1016/j.cortex.2008.04.001

Gootjes, L., Bouma, A., Van Strien, J. W., Van Schijndel, R., Barkhof, F., \& Scheltens, P. (2006). Corpus callosum size correlates with asymmetric performance on a dichotic listening task in healthy aging but not in Alzheimer's disease. Neuropsychologia, 44(2), 208-217. doi:10.1016/j.neuropsychologia.2005.05.002

Hasan, K. M., Ewing-Cobbs, L., Kramer, L. A., Fletcher, J. M., \& Narayana, P. A. (2008). Diffusion tensor quantification of the macrostructure and microstructure of human midsagittal corpus callosum across the lifespan. NMR Biomed, 21(10), 1094-1101. doi:10.1002/nbm.1286

Hasan, K. M., Kamali, A., Iftikhar, A., Kramer, L. A., Papanicolaou, A. C., Fletcher, J. M., \& Ewing-Cobbs, L. (2009). Diffusion tensor tractography quantification of the human corpus callosum fiber pathways across the lifespan. Brain research, 1249, 91-100.

Hasan, K. M., Kamali, A., Kramer, L. A., Papnicolaou, A. C., Fletcher, J. M., \& Ewing-Cobbs, L. (2008). Diffusion tensor quantification of the human midsagittal corpus callosum subdivisions across the lifespan. Brain Res, 1227, 52-67. doi:10.1016/j.brainres.2008.06.030

Hayes, A. F. (2009). Beyond Baron and Kenny: Statistical mediation analysis in the new millennium. Communication monographs, 76(4), 408-420.

Hinkley, L. B., Marco, E. J., Brown, E. G., Bukshpun, P., Gold, J., Hill, S., . . Nagarajan, S. S. (2016). The Contribution of the Corpus Callosum to Language Lateralization. J Neurosci, 36(16), 4522-4533. doi:10.1523/JNEUROSCI.3850-14.2016

Hofer, S., \& Frahm, J. (2006). Topography of the human corpus callosum revisited--comprehensive fiber tractography using diffusion tensor magnetic resonance imaging. Neuroimage, 32(3), 989-994. doi:10.1016/j.neuroimage.2006.05.044

Holloway, R. L., \& de Lacoste, M. C. (1986). Sexual dimorphism in the human corpus callosum: an extension and replication study. Hum Neurobiol, 5(2), 87-91. Retrieved from https://www.ncbi.nlm.nih.gov/pubmed/3733478

Hou, J., \& Pakkenberg, B. (2012). Age-related degeneration of corpus callosum in the $90+$ years measured with stereology. Neurobiol Aging, 33(5), 1009 e1001-1009. doi:10.1016/j.neurobiolaging.2011.10.017

Huster, R. J., Westerhausen, R., \& Herrmann, C. S. (2011). Sex differences in cognitive control are associated with midcingulate and callosal morphology. Brain Struct Funct, 215(3-4), 225-235. doi:10.1007/s00429-010-0289-2

Innocenti, G. M., \& Price, D. J. (2005). Exuberance in the development of cortical networks. Nat Rev Neurosci, 6(12), 955-965. doi:10.1038/nrn1790

Jancke, L., Staiger, J. F., Schlaug, G., Huang, Y., \& Steinmetz, H. (1997). The relationship between corpus callosum size and forebrain volume. Cereb Cortex, 7(1), 48-56. Retrieved from http://www.ncbi.nlm.nih.gov/pubmed/9023431

Kennedy, K. M., \& Raz, N. (2009). Aging white matter and cognition: differential effects of regional variations in diffusion properties on memory, executive functions, and speed. Neuropsychologia, 47(3), 916-927. doi:10.1016/j.neuropsychologia.2009.01.001

Krogsrud, S. K., Fjell, A. M., Tamnes, C. K., Grydeland, H., Mork, L., Due-Tonnessen, P., . . Walhovd, K. B. (2016). Changes in white matter microstructure in the developing brain-A longitudinal diffusion tensor imaging study of children from 4 to 11years of age. Neuroimage, 124(Pt A), 473-486. doi:10.1016/j.neuroimage.2015.09.017

LaMantia, A. S., \& Rakic, P. (1990). Axon overproduction and elimination in the corpus callosum of the developing rhesus monkey. $J$ Neurosci, 10(7), 2156-2175. Retrieved from https://www.ncbi.nlm.nih.gov/pubmed/2376772 
Lebel, C., Caverhill-Godkewitsch, S., \& Beaulieu, C. (2010). Age-related regional variations of the corpus callosum identified by diffusion tensor tractography. Neuroimage, 52(1), 20-31. doi:10.1016/j.neuroimage.2010.03.072

Lebel, C., Walker, L., Leemans, A., Phillips, L., \& Beaulieu, C. (2008). Microstructural maturation of the human brain from childhood to adulthood. Neuroimage, 40(3), 1044-1055. doi:10.1016/j.neuroimage.2007.12.053

Luders, E., Cherbuin, N., Thompson, P. M., Gutman, B., Anstey, K. J., Sachdev, P., \& Toga, A. W. (2010). When more is less: associations between corpus callosum size and handedness lateralization. Neuroimage, 52(1), 43-49. doi:10.1016/j.neuroimage.2010.04.016

Luders, E., Narr, K. L., Bilder, R. M., Thompson, P. M., Szeszko, P. R., Hamilton, L., \& Toga, A. W. (2007). Positive correlations between corpus callosum thickness and intelligence. Neuroimage, 37(4), 1457-1464. doi:10.1016/j.neuroimage.2007.06.028

Luders, E., Narr, K. L., Zaidel, E., Thompson, P. M., \& Toga, A. W. (2006). Gender effects on callosal thickness in scaled and unscaled space. Neuroreport, 17(11), 1103-1106. doi:10.1097/01.wnr.0000227987.77304.cc

Luders, E., Thompson, P. M., \& Toga, A. W. (2010). The development of the corpus callosum in the healthy human brain. $J$ Neurosci, 30(33), 10985-10990. doi:10.1523/JNEUROSCI.5122-09.2010

Madden, D. J., Bennett, I. J., \& Song, A. W. (2009). Cerebral white matter integrity and cognitive aging: contributions from diffusion tensor imaging. Neuropsychol Rev, 19(4), 415-435. doi:10.1007/s11065-009-9113-2

Maechler, M. (2019). "Finding Groups in Data": Cluster Analysis Extended Rousseeuw et al. R Packag. version 2.1.

Neisser, U. (1998). Introduction: Rising test scores and what they mean. American Scientist, 85(5), 440-447.

Ocklenburg, S., \& Güntürkün, O. (2018). The lateralized brain: The neuroscience and evolution of hemispheric asymmetries. London, UK: Academic Press.

Penke, L., Maniega, S. M., Bastin, M., Hernandez, M. V., Murray, C., Royle, N., . . Deary, I. (2012). Brain white matter tract integrity as a neural foundation for general intelligence. Molecular psychiatry, 17(10), 1026-1030.

Penke, L., Munoz Maniega, S., Houlihan, L. M., Murray, C., Gow, A. J., Clayden, J. D., . . Deary, I. J. (2010). White matter integrity in the splenium of the corpus callosum is related to successful cognitive aging and partly mediates the protective effect of an ancestral polymorphism in ADRB2. Behav Genet, 40(2), 146-156. doi:10.1007/s10519-009-9318-4

Peters, A., \& Sethares, C. (2002). Aging and the myelinated fibers in prefrontal cortex and corpus callosum of the monkey. Journal of Comparative Neurology, 442(3), 277-291. doi:10.1002/cne.10099

Prendergast, D. M., Ardekani, B., Ikuta, T., John, M., Peters, B., DeRosse, P., . . Szeszko, P. R. (2015). Age and sex effects on corpus callosum morphology across the lifespan. Hum Brain Mapp, 36(7), 26912702. doi:10.1002/hbm. 22800

Pujol, J., Vendrell, P., Junque, C., Marti-Vilalta, J. L., \& Capdevila, A. (1993). When does human brain development end? Evidence of corpus callosum growth up to adulthood. Ann Neurol, 34(1), 71-75. doi:10.1002/ana.410340113

Putnam, M. C., Wig, G. S., Grafton, S. T., Kelley, W. M., \& Gazzaniga, M. S. (2008). Structural organization of the corpus callosum predicts the extent and impact of cortical activity in the nondominant hemisphere. $J$ Neurosci, 28(11), 2912-2918. doi:10.1523/JNEUROSCI.2295-07.2008

Rajapakse, J. C., Giedd, J. N., Rumsey, J. M., Vaituzis, A. C., Hamburger, S. D., \& Rapoport, J. L. (1996). Regional MRI measurements of the corpus callosum: a methodological and developmental study. Brain Dev, 18(5), 379-388. doi:10.1016/0387-7604(96)00034-4

Rakic, P., \& Yakovlev, P. I. (1968). Development of the corpus callosum and cavum septi in man. J Comp Neurol, 132(1), 45-72. doi:10.1002/cne.901320103

Rauch, R. A., \& Jinkins, J. R. (1994). Analysis of cross-sectional area measurements of the corpus callosum adjusted for brain size in male and female subjects from childhood to adulthood. Behav Brain Res, 64(1-2), 65-78. Retrieved from http://www.ncbi.nlm.nih.gov/pubmed/7840893

Raz, N., Ghisletta, P., Rodrigue, K. M., Kennedy, K. M., \& Lindenberger, U. (2010). Trajectories of brain aging in middle-aged and older adults: regional and individual differences. Neuroimage, 51(2), 501511. doi:10.1016/j.neuroimage.2010.03.020

Riise, J., \& Pakkenberg, B. (2011). Stereological estimation of the total number of myelinated callosal fibers in human subjects. J Anat, 218(3), 277-284. doi:10.1111/j.1469-7580.2010.01333.x 
Rosseel, Y. (2012). Lavaan: An R package for structural equation modeling and more. Version 0.5-12 (BETA). Journal of statistical software, 48(2), 1-36.

Salami, A., Eriksson, J., Nilsson, L. G., \& Nyberg, L. (2012). Age-related white matter microstructural differences partly mediate age-related decline in processing speed but not cognition. Biochim Biophys Acta, 1822(3), 408-415. doi:10.1016/j.bbadis.2011.09.001

Salat, D., Ward, A., Kaye, J. A., \& Janowsky, J. S. (1997). Sex differences in the corpus callosum with aging. Neurobiol Aging, 18(2), 191-197. doi:10.1016/s0197-4580(97)00014-6

Salat, D. H., Tuch, D. S., Greve, D. N., van der Kouwe, A. J., Hevelone, N. D., Zaleta, A. K., . . Dale, A. M. (2005). Age-related alterations in white matter microstructure measured by diffusion tensor imaging. Neurobiol Aging, 26(8), 1215-1227. doi:10.1016/j.neurobiolaging.2004.09.017

Scally, B., Burke, M. R., Bunce, D., \& Delvenne, J. F. (2018). Visual and visuomotor interhemispheric transfer time in older adults. Neurobiol Aging, 65, 69-76. doi:10.1016/j.neurobiolaging.2018.01.005

Schmahmann, J. D., \& Pandya, D. N. (2006). Fiber pathways of the brain. Oxford, UK: Oxford University Press.

Schulte, T., Müller-Oehring, E., Salo, R., Pfefferbaum, A., \& Sullivan, E. (2006). Callosal involvement in a lateralized stroop task in alcoholic and healthy subjects. Neuropsychology, 20(6), 727.

Skumlien, M., Sederevicius, D., Fjell, A. M., Walhovd, K. B., \& Westerhausen, R. (2018). Parallel but independent reduction of emotional awareness and corpus callosum connectivity in older age. PLoS One, 13(12), e0209915.

Smith, R. J. (2005). Relative size versus controlling for size. Current Anthropology, 46(2), 249-273.

Steinmann, S., Amselberg, R., Cheng, B., Thomalla, G., Engel, A. K., Leicht, G., \& Mulert, C. (2018). The role of functional and structural interhemispheric auditory connectivity for language lateralization A combined EEG and DTI study. Sci Rep, 8(1), 15428. doi:10.1038/s41598-018-33586-6

Sullivan, E. V., Adalsteinsson, E., \& Pfefferbaum, A. (2006). Selective age-related degradation of anterior callosal fiber bundles quantified in vivo with fiber tracking. Cereb Cortex, 16(7), 1030-1039. doi:10.1093/cercor/bhj045

Sullivan, E. V., Rohlfing, T., \& Pfefferbaum, A. (2010). Quantitative fiber tracking of lateral and interhemispheric white matter systems in normal aging: relations to timed performance. Neurobiol Aging, 31(3), 464-481. doi:10.1016/j.neurobiolaging.2008.04.007

Tamnes, C. K., Ostby, Y., Fjell, A. M., Westlye, L. T., Due-Tonnessen, P., \& Walhovd, K. B. (2010). Brain maturation in adolescence and young adulthood: regional age-related changes in cortical thickness and white matter volume and microstructure. Cereb Cortex, 20(3), 534-548. doi:10.1093/cercor/bhp118

Tamnes, C. K., Walhovd, K. B., Dale, A. M., Ostby, Y., Grydeland, H., Richardson, G., . . Alzheimer's Disease Neuroimaging, I. (2013). Brain development and aging: overlapping and unique patterns of change. Neuroimage, 68, 63-74. doi:10.1016/j.neuroimage.2012.11.039

Tanaka-Arakawa, M. M., Matsui, M., Tanaka, C., Uematsu, A., Uda, S., Miura, K., . . Noguchi, K. (2015). Developmental changes in the corpus callosum from infancy to early adulthood: a structural magnetic resonance imaging study. PLoS One, 10(3), e0118760. doi:10.1371/journal.pone. 0118760

Tang, C., Eaves, E., Ng, J., Carpenter, D., Mai, X., Schroeder, D., . . Haier, R. (2010). Brain networks for working memory and factors of intelligence assessed in males and females with fMRI and DTI. Intelligence, 38(3), 293-303.

Thiel, A., Schumacher, B., Wienhard, K., Gairing, S., Kracht, L. W., Wagner, R., .. Heiss, W. D. (2006). Direct demonstration of transcallosal disinhibition in language networks. J Cereb Blood Flow Metab, 26(9), 1122-1127. doi:10.1038/sj.jcbfm.9600350

Thompson, P. M., Giedd, J. N., Woods, R. P., MacDonald, D., Evans, A. C., \& Toga, A. W. (2000). Growth patterns in the developing brain detected by using continuum mechanical tensor maps. Nature, 404(6774), 190-193. doi:10.1038/35004593

Tomaiuolo, F., Voci, L., Bresci, M., Cozza, S., Posteraro, F., Oliva, M., \& Doricchi, F. (2010). Selective visual neglect in right brain damaged patients with splenial interhemispheric disconnection. Exp Brain Res, 206(2), 209-217. doi:10.1007/s00221-010-2230-6

Vannucci, R. C., Barron, T. F., \& Vannucci, S. J. (2017). Development of the Corpus Callosum: An MRI Study. Dev Neurosci, 39(1-4), 97-106. doi:10.1159/000453031

Wechsler, D. (1999). Wechsler Abbreviated Scale of Intelligence (WASI). San Antonio, USA: The Psychological Corporation. 
Wechsler, D. (2002). Wechsler Preschool and Primary Scale of Intelligence -Third Edition (WPPSI-III). San Antonio, USA: The Psychological Corporation.

Westerhausen, R., Fjell, A. M., Krogsrud, S. K., Rohani, D. A., Skranes, J. S., Haberg, A. K., \& Walhovd, K. B. (2016). Selective increase in posterior corpus callosum thickness between the age of 4 and 11years. Neuroimage, 139, 17-25. doi:10.1016/j.neuroimage.2016.06.008

Westerhausen, R., Friesen, C. M., Rohani, D. A., Krogsrud, S. K., Tamnes, C. K., Skranes, J. S., . . . Walhovd, K. B. (2018). The corpus callosum as anatomical marker of intelligence? A critical examination in a large-scale developmental study. Brain Struct Funct, 223(1), 285-296. doi:10.1007/s00429-017-1493-0

Westerhausen, R., Gruner, R., Specht, K., \& Hugdahl, K. (2009). Functional relevance of interindividual differences in temporal lobe callosal pathways: a DTI tractography study. Cereb Cortex, 19(6), 1322-1329. doi:10.1093/cercor/bhn173

Westerhausen, R., \& Karud, C. M. R. (2018). Callosotomy affects performance IQ: A meta-analysis of individual participant data. Neurosci Lett, 665, 43-47. doi:10.1016/j.neulet.2017.11.040

Westerhausen, R., Kreuder, F., Woerner, W., Huster, R. J., Smit, C. M., Schweiger, E., \& Wittling, W. (2006). Interhemispheric transfer time and structural properties of the corpus callosum. Neuroscience letters, 409(2), 140-145.

Westerhausen, R., Luders, E., Specht, K., Ofte, S. H., Toga, A. W., Thompson, P. M., . . Hugdahl, K. (2011). Structural and functional reorganization of the corpus callosum between the age of 6 and 8 years. Cereb Cortex, 21(5), 1012-1017. doi:10.1093/cercor/bhq165

Westlye, L. T., Walhovd, K. B., Dale, A. M., Bjornerud, A., Due-Tonnessen, P., Engvig, A., . . Fjell, A. M. (2010). Life-span changes of the human brain white matter: diffusion tensor imaging (DTI) and volumetry. Cereb Cortex, 20(9), 2055-2068. doi:10.1093/cercor/bhp280

Whitford, T. J., Kubicki, M., Ghorashi, S., Schneiderman, J. S., Hawley, K. J., McCarley, R. W., ... Spencer, K. M. (2011). Predicting inter-hemispheric transfer time from the diffusion properties of the corpus callosum in healthy individuals and schizophrenia patients: a combined ERP and DTI study. Neuroimage, 54(3), 2318-2329. doi:10.1016/j.neuroimage.2010.10.048

Witelson, S. F. (1989). Hand and sex differences in the isthmus and genu of the human corpus callosum. A postmortem morphological study. Brain, 112 ( Pt 3), 799-835. Retrieved from http://www.ncbi.nlm.nih.gov/pubmed/2731030

Wood, S. N. (2017). Generalized additive models: an introduction with R. Boca Raton, FL, USA: Chapman and Hall/CRC.

Yeatman, J. D., Wandell, B. A., \& Mezer, A. A. (2014). Lifespan maturation and degeneration of human brain white matter. Nat Commun, 5, 4932. doi:10.1038/ncomms5932

Zahr, N. M., Rohlfing, T., Pfefferbaum, A., \& Sullivan, E. V. (2009). Problem solving, working memory, and motor correlates of association and commissural fiber bundles in normal aging: a quantitative fiber tracking study. Neuroimage, 44(3), 1050-1062. doi:10.1016/j.neuroimage.2008.09.046 


\section{Supplement}

Table S1. Segment-wise modelling lifespan trajectories of relative callosal thickness

For each segment, a GAMM analysis was set-up using the "mgcv" package (Wood, 2017) with the dependent variable relative callosal thickness (thickness/FBV ${ }^{0.333}$ ). The model contained Age (smoothed with cubic regression splines and $\mathrm{k}=10 \mathrm{knots}$ ) in the fixed-effect part of the model, together with Sex and Scanner as covariates of non-interest. The random effects part of the model contained a participant identifier (considering intercept differences). The model fitting was done using restricted maximum likelihood (REML) estimations. The number of data points included was $\mathrm{N}=1867$ between the age of 4.1 and 93.3 years. An overview of the results can be found in the table below.

Table S1. Results of GAMM modelling by segment

\begin{tabular}{|c|c|c|c|c|c|c|c|c|}
\hline \multirow[b]{2}{*}{$\operatorname{Segm}^{\mathrm{a}}$} & \multirow[b]{2}{*}{ Cluster $^{\mathrm{b}}$} & \multirow{2}{*}{$\begin{array}{r}\text { Model } \\
\mathrm{R}_{\mathrm{adj}}{ }^{2} \\
\end{array}$} & \multicolumn{2}{|c|}{ Effect of Age } & \multirow[b]{2}{*}{$\mathrm{p}(\mathrm{FDR})^{\mathrm{c}}$} & \multirow[b]{2}{*}{$\omega^{2 d}$} & \multicolumn{2}{|c|}{ Covariates (p-values) ${ }^{\mathrm{c}}$} \\
\hline & & & $\mathrm{F}$ & edf & & & Sex & Scanner \\
\hline 1 & 1 & 0.08 & 57.03 & 1.73 & $<0.001^{\mathrm{g}}$ & 0.05 & 1.000 & 1.000 \\
\hline 2 & 1 & 0.14 & 61.27 & 2.67 & $<0.001$ & 0.08 & 0.782 & 1.000 \\
\hline 3 & 1 & 0.14 & 50.35 & 3.01 & $<0.001$ & 0.07 & 0.133 & 1.000 \\
\hline 4 & 1 & 0.11 & 44.20 & 2.88 & $<0.001$ & 0.06 & 0.603 & 1.000 \\
\hline 5 & 1 & 0.11 & 113.33 & 1.00 & $<0.001$ & 0.06 & 0.603 & 1.000 \\
\hline 6 & $3^{\mathrm{e}}$ & 0.10 & 30.92 & 3.67 & $<0.001$ & 0.06 & 1.000 & 0.681 \\
\hline 7 & $2 a^{f}$ & 0.12 & 39.08 & 4.65 & $<0.001$ & 0.09 & 1.000 & $<0.001$ \\
\hline 8 & $2 \mathrm{a}$ & 0.13 & 43.33 & 5.23 & $<0.001$ & 0.11 & 0.782 & $<0.001$ \\
\hline 9 & $2 \mathrm{a}$ & 0.13 & 41.68 & 5.11 & $<0.001$ & 0.10 & 0.059 & $<0.001$ \\
\hline 10 & $2 \mathrm{a}$ & 0.13 & 39.11 & 4.81 & $<0.001$ & 0.09 & 0.025 & $<0.001$ \\
\hline 11 & $2 a$ & 0.12 & 41.11 & 4.73 & $<0.001$ & 0.09 & 0.059 & $<0.001$ \\
\hline 12 & $2 a$ & 0.12 & 42.40 & 4.81 & $<0.001$ & 0.10 & 0.548 & $<0.001$ \\
\hline 13 & $2 a$ & 0.13 & 43.83 & 5.00 & $<0.001$ & 0.10 & 0.782 & $<0.001$ \\
\hline 14 & 3 & 0.15 & 50.44 & 4.45 & $<0.001$ & 0.11 & 1.000 & $<0.001$ \\
\hline 15 & 3 & 0.16 & 53.74 & 4.55 & $<0.001$ & 0.11 & 0.808 & $<0.001$ \\
\hline 16 & 3 & 0.16 & 55.47 & 4.58 & $<0.001$ & 0.12 & 1.000 & $<0.001$ \\
\hline 17 & 3 & 0.15 & 50.40 & 4.60 & $<0.001$ & 0.11 & 1.000 & $<0.001$ \\
\hline 18 & 3 & 0.14 & 49.83 & 4.40 & $<0.001$ & 0.10 & 1.000 & $<0.001$ \\
\hline 19 & 3 & 0.14 & 48.78 & 4.54 & $<0.001$ & 0.10 & 1.000 & $<0.001$ \\
\hline 20 & 3 & 0.13 & 45.64 & 4.29 & $<0.001$ & 0.09 & 1.000 & 0.004 \\
\hline 21 & 3 & 0.12 & 41.57 & 4.40 & $<0.001$ & 0.09 & 1.000 & 0.022 \\
\hline 22 & 3 & 0.12 & 41.47 & 4.50 & $<0.001$ & 0.09 & 1.000 & $<0.001$ \\
\hline 23 & 3 & 0.12 & 36.23 & 4.95 & $<0.001$ & 0.09 & 1.000 & 0.001 \\
\hline 24 & 3 & 0.11 & 36.16 & 4.92 & $<0.001$ & 0.08 & 1.000 & $\mathbf{0 . 0 0 3}$ \\
\hline 25 & 3 & 0.11 & 33.70 & 4.77 & $<0.001$ & 0.08 & 1.000 & $<0.001$ \\
\hline 26 & 3 & 0.11 & 33.09 & 4.80 & $<0.001$ & 0.08 & 1.000 & 0.011 \\
\hline 27 & 3 & 0.10 & 32.25 & 4.44 & $<0.001$ & 0.07 & 1.000 & 0.139 \\
\hline 28 & 3 & 0.10 & 30.74 & 4.35 & $<0.001$ & 0.06 & 1.000 & $<0.001$ \\
\hline 29 & 3 & 0.10 & 30.61 & 4.65 & $<0.001$ & 0.07 & 1.000 & $<0.001$ \\
\hline
\end{tabular}




\begin{tabular}{|c|c|c|c|c|c|c|c|c|}
\hline 30 & 3 & 0.09 & 27.09 & 4.69 & $<0.001$ & 0.06 & 1.000 & $<0.001$ \\
\hline 31 & 3 & 0.09 & 25.09 & 4.71 & $<0.001$ & 0.06 & 1.000 & $<0.001$ \\
\hline 32 & 3 & 0.08 & 24.90 & 4.84 & $<0.001$ & 0.06 & 1.000 & 0.015 \\
\hline 33 & 3 & 0.07 & 21.15 & 4.92 & $<0.001$ & 0.05 & 1.000 & 0.022 \\
\hline 34 & 3 & 0.07 & 21.30 & 4.73 & $<0.001$ & 0.05 & 1.000 & 0.013 \\
\hline 35 & 3 & 0.07 & 21.26 & 4.58 & $<0.001$ & 0.05 & 1.000 & 0.030 \\
\hline 36 & 3 & 0.06 & 21.20 & 4.36 & $<0.001$ & 0.05 & 1.000 & 0.874 \\
\hline 37 & 3 & 0.06 & 17.65 & 4.38 & $<0.001$ & 0.04 & 1.000 & 0.034 \\
\hline 38 & 3 & 0.05 & 14.93 & 4.17 & $<0.001$ & 0.03 & 1.000 & 0.024 \\
\hline 39 & 3 & 0.05 & 12.84 & 5.11 & $<0.001$ & 0.03 & 1.000 & 0.874 \\
\hline 40 & 3 & 0.07 & 14.26 & 5.14 & $<0.001$ & 0.04 & 1.000 & 0.804 \\
\hline 41 & 3 & 0.10 & 21.81 & 5.09 & $<0.001$ & 0.05 & 1.000 & 0.250 \\
\hline 42 & 3 & 0.11 & 25.13 & 4.89 & $<0.001$ & 0.06 & 1.000 & 0.008 \\
\hline 43 & 3 & 0.12 & 26.00 & 5.13 & $<0.001$ & 0.06 & 0.472 & 0.002 \\
\hline 44 & 3 & 0.13 & 30.74 & 4.83 & $<0.001$ & 0.07 & 0.485 & $<0.001$ \\
\hline 45 & $2 p^{f}$ & 0.13 & 33.29 & 5.07 & $<0.001$ & 0.08 & 0.485 & 0.008 \\
\hline 46 & $2 p$ & 0.12 & 30.08 & 5.85 & $<0.001$ & 0.08 & 0.526 & $<0.001$ \\
\hline 47 & $2 p$ & 0.12 & 33.93 & 5.71 & $<0.001$ & 0.09 & 0.485 & $<0.001$ \\
\hline 48 & 4 & 0.13 & 38.59 & 6.40 & $<0.001$ & 0.11 & 0.203 & $<0.001$ \\
\hline 49 & 4 & 0.13 & 41.05 & 6.40 & $<0.001$ & 0.12 & 0.188 & $<0.001$ \\
\hline 50 & 4 & 0.15 & 45.02 & 6.26 & $<0.001$ & 0.13 & 0.171 & $<0.001$ \\
\hline 51 & 4 & 0.16 & 49.90 & 6.29 & $<0.001$ & 0.14 & 0.104 & $<0.001$ \\
\hline 52 & 4 & 0.17 & 53.69 & 6.46 & $<0.001$ & 0.15 & 0.028 & $<0.001$ \\
\hline 53 & 4 & 0.16 & 60.58 & 6.35 & $<0.001$ & 0.17 & 0.028 & $<0.001$ \\
\hline 54 & 4 & 0.15 & 59.20 & 6.35 & $<0.001$ & 0.17 & 0.028 & $<0.001$ \\
\hline 55 & 4 & 0.14 & 52.07 & 6.22 & $<0.001$ & 0.15 & 0.086 & $<0.001$ \\
\hline 56 & 5 & 0.18 & 60.85 & 6.57 & $<0.001$ & 0.17 & 0.171 & $<0.001$ \\
\hline 57 & 5 & 0.25 & 69.46 & 6.73 & $<0.001$ & 0.20 & 0.186 & $<0.001$ \\
\hline 58 & 5 & 0.30 & 74.31 & 6.50 & $<0.001$ & 0.20 & 0.290 & $<0.001$ \\
\hline 59 & 5 & 0.28 & 68.03 & 6.34 & $<0.001$ & 0.19 & 0.934 & 0.005 \\
\hline 60 & $3^{\mathrm{e}}$ & 0.25 & 64.12 & 5.41 & $<0.001$ & 0.15 & 1.000 & 0.378 \\
\hline
\end{tabular}

Notes. (a) Segments are numbered ordered from anterior $(1=$ rostrum) to posterior (60 =splenium) segments; (b) indicates the cluster membership according to the subsequent PAM analysis (see main text), (c) All p-values are adjusted to achieve a false-discovery rate of FDR = 0.05; (d) $\omega^{2}=$ proportion explained variance of relative callosal thickness by Age; (e) clusters 6 and 60 were originally assigned to cluster 3 but were excluded from further analysis steps as they are spatial outliers, (f) segments marked as $2 a$ (anterior) and $2 p$ (posterior) were split due to spatial distance, but were originally assigned to the same cluster by the clustering algorithm, (g) significant effects, i.e. $p(F D R)<0.05$, are written bold 


\section{Table S2. Testing requirements for mediation analysis}

Following Hayes (2009), a mediation analysis may be considered meaningful, only if the associations of the predictor and mediator (condition 1), and mediator and dependent variable (condition 2) are present. Table S2 shows for each combination callosal cluster and cognitive test, the results of linear regression analyses testing these conditions. Only if both analyses were significant, the mediation analysis was conducted (as indicated in the column "Included". Details on sample selection and calculation of the change score can be found in the main text.

Table S2. Testing of requirements for mediation analysis, i.e. that change in Age ( $\triangle \mathrm{AGE}$ ) predicts the mediator change relative callosal thickness $(\triangle \mathrm{RCT})$, and that $\triangle \mathrm{RCT}$ predicts change in cognition $(\triangle \mathrm{COG})$

\begin{tabular}{|c|c|c|c|c|c|c|c|c|c|c|}
\hline \multirow{2}{*}{ Block $^{\mathrm{a}}$} & \multirow{2}{*}{ Cluster } & \multicolumn{4}{|c|}{ Condition 1: $\triangle \mathrm{AGE} \rightarrow \Delta \mathrm{RCT}$} & \multicolumn{4}{|c|}{ Condition 2: $\Delta \mathrm{RCT} \rightarrow \Delta \mathrm{COG}$} & \multirow{2}{*}{ Incl. ${ }^{c}$} \\
\hline & & slope & t-value ${ }^{b}$ & $\mathrm{p}$ & $\omega^{2}$ & slope & t-value ${ }^{b}$ & $\mathrm{P}$ & $\omega^{2}$ & \\
\hline \multirow[t]{6}{*}{ MR } & 1 & -0.004 & -0.593 & 0.554 & $<0.01$ & 0.369 & 1.257 & 0.209 & $<0.01$ & No \\
\hline & $2 \mathrm{a}$ & -0.006 & -1.325 & 0.186 & $<0.01$ & -0.196 & -0.479 & 0.632 & $<0.01$ & No \\
\hline & 3 & -0.008 & -4.084 & $<0.001$ & 0.03 & 1.275 & 1.443 & 0.150 & $<0.01$ & No \\
\hline & $2 p$ & -0.012 & -3.346 & 0.001 & 0.02 & 1.541 & 3.034 & 0.003 & 0.02 & Yes \\
\hline & 4 & -0.019 & -6.409 & $<0.001$ & 0.08 & 2.674 & 4.754 & $<0.001$ & 0.05 & Yes \\
\hline & 5 & -0.018 & -4.959 & $<0.001$ & 0.05 & 0.560 & 1.185 & 0.237 & $<0.01$ & No \\
\hline \multirow[t]{6}{*}{ VOC } & 1 & -0.004 & -0.593 & 0.554 & $<0.01$ & 1.133 & 2.752 & 0.006 & 0.02 & No \\
\hline & $2 a$ & -0.006 & -1.325 & 0.186 & $<0.01$ & 0.047 & 0.082 & 0.935 & $<0.01$ & No \\
\hline & 3 & -0.008 & -4.084 & $<0.001$ & 0.03 & 4.223 & 3.441 & 0.001 & 0.02 & Yes \\
\hline & $2 p$ & -0.012 & -3.346 & 0.001 & 0.02 & 2.706 & 3.823 & $<0.001$ & 0.03 & Yes \\
\hline & 4 & -0.019 & -6.409 & $<0.001$ & 0.08 & 4.134 & 5.265 & $<0.001$ & 0.06 & Yes \\
\hline & 5 & -0.018 & -4.959 & $<0.001$ & 0.05 & 1.861 & 2.827 & 0.005 & 0.02 & Yes \\
\hline
\end{tabular}

Notes. (a) In Block A, $\triangle C O G$ is the change in Matrix Reasoning (MR) raw score, in Block B change in Vocabulary (VOC) raw score; (b) degrees of freedom for each analysis was $d f=477 . ;$ (c) Included in change mediation analysis 
Figure S1. Results of cluster analysis using callosal thickness relative to white-matter volume (WMV) as dependant variable

Initial PAM clustering of the callosal segments based on the first derivative of the lifespan trajectories of relative thickness (i.e., thickness/WMV ${ }^{0.333}$ ). Panel A shows the silhouette coefficient for cluster solutions between 2 to 10 clusters. A 4-cluster solution was selected as it showed a slight peak of the silhouette coefficient, and was followed by a substantial drop for a 5-cluster solution. Panel B shows the segment-wise color-coded topography of the clusters within the corpus callosum for the present solution. Panel C shows the lifespan trajectories of mean relative thickness (top row) and their first derivatives (bottom row) for each segment (different lines) per cluster. Bold black lines in each graph represent the mean trajectory within the cluster. Of note, positive values in the derivative plots indicate an over-proportional growth of relative thickness while negative values indicate an over-proportional decline.
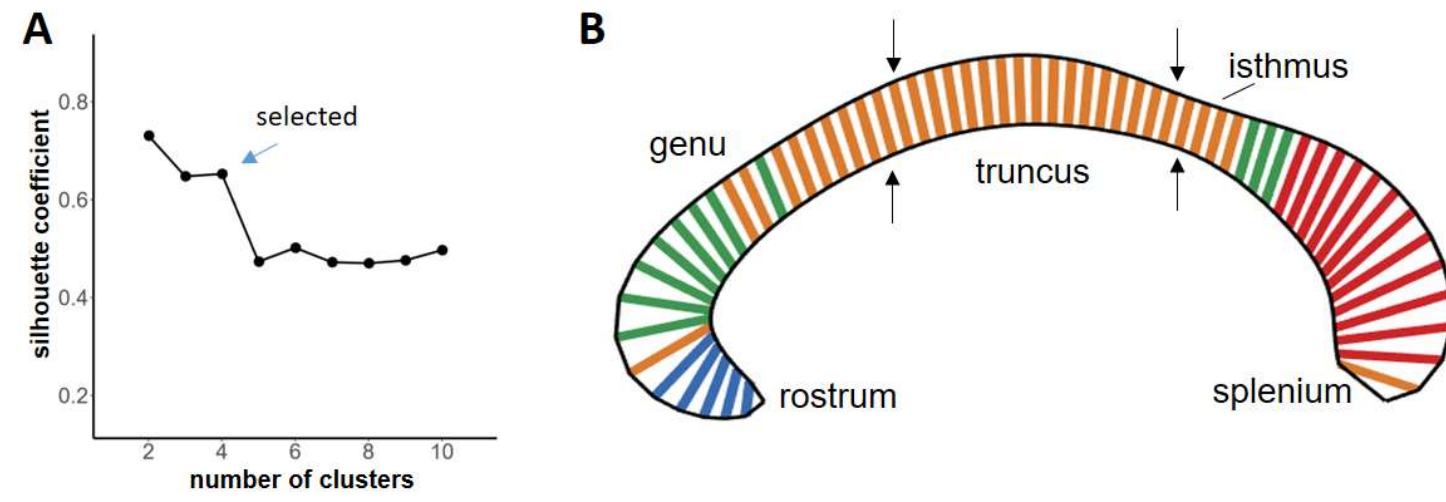

C

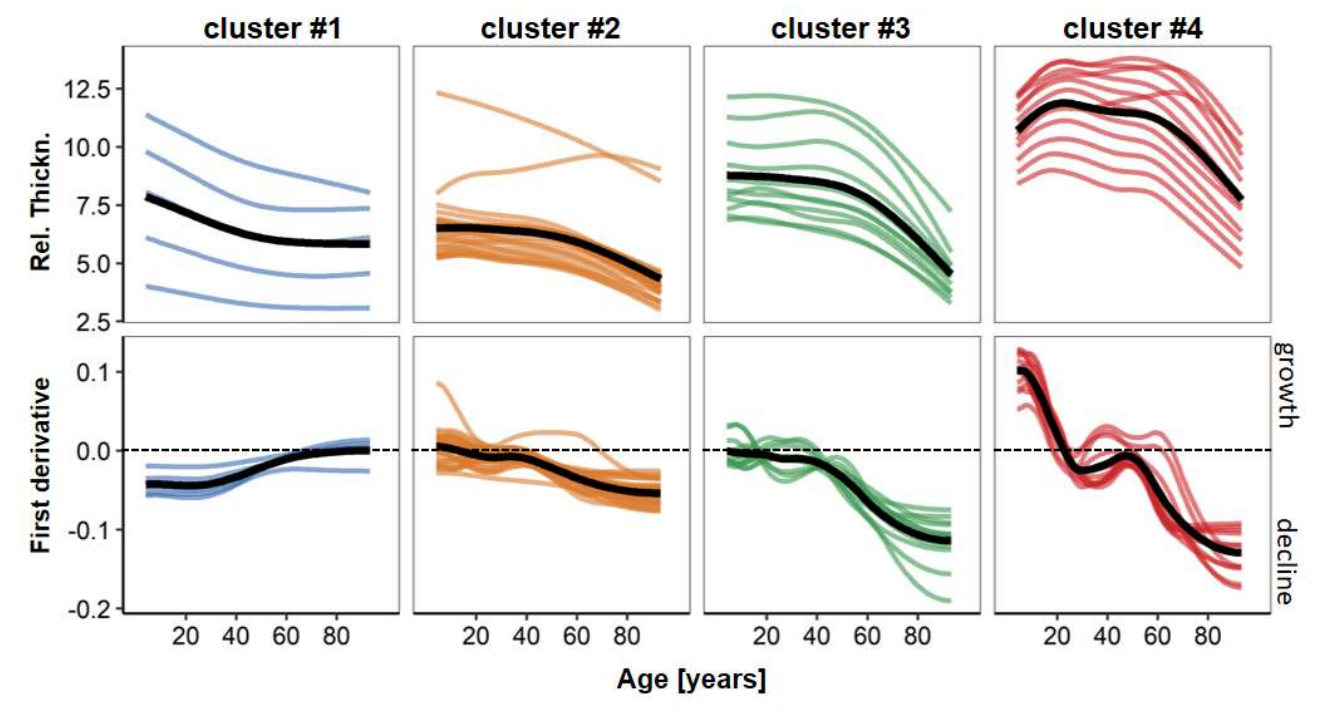


Table S3. Results of cluster analysis using thickness relative to white-matter volume trajectories (descriptive statistics)

Table S3. Descriptive statistics for the clusters as identified in Fig. S1. ${ }^{\text {a }}$

\begin{tabular}{|c|c|c|c|c|c|c|c|}
\hline Cluster $^{\mathrm{b}}$ & $\begin{array}{c}\text { Number of } \\
\text { segments }\end{array}$ & mean edf & s.d. edf & min edf & max edf & EoG $^{\mathrm{c}}$ & BoD $^{\mathrm{d}}$ \\
\hline 1 & 5 & 3.03 & 0.30 & 2.62 & 3.30 & n.a. ${ }^{\mathrm{e}}$ & n.a.$^{\mathrm{e}}$ \\
\hline $3 \mathrm{a}$ & 7 & 4.61 & 0.32 & 4.10 & 4.99 & n.a. & 41.8 \\
\hline 2 & 30 & 4.02 & 0.25 & 3.35 & 4.36 & n.a. & 31.5 \\
\hline $3 \mathrm{p}$ & 3 & 5.09 & 0.52 & 4.51 & 5.51 & n.a. & 21.6 \\
\hline 4 & 12 & 6.10 & 0.16 & 5.78 & 6.43 & 21.2 & 53.0 \\
\hline
\end{tabular}

Notes. (a) the three outlier segments visible in Fig. S1 have been excluded., (b) ordered from anterior (rostrum) to posterior (splenium), (c) EoG = end of growth, (d) BoD = beginning of decline, (e) not available as it falls before the studied age period

\section{References Supplement}

Hayes, A. F. (2009). Beyond Baron and Kenny: Statistical mediation analysis in the new millennium. Communication monographs, 76(4), 408-420.

Wood, S. N. (2017). Generalized additive models: an introduction with R. Boca Raton, FL, USA:

Chapman and Hall/CRC. 